\title{
Insect-Inspired Mechanical Resilience for Multicopters
}

\author{
S. Mintchev, Member, IEEE, S. de Rivaz and D. Floreano, Senior Member, IEEE
}

\begin{abstract}
The ease of use and versatility of drones has contributed to their deployment in several fields, from entertainment to search and rescue. However, drones remain vulnerable to collisions due to pilot mistakes or various system failures. This paper presents a bioinspired strategy for the design of quadcopters resilient to collisions. Abstracting the biomechanical strategy of collision resilient insects' wings, the quadcopter has a dual-stiffness frame that rigidly withstands aerodynamic loads within the flight envelope, but can soften and fold during a collision to avoid damage. The dual-stiffness frame works in synergy with specific energy absorbing materials that protect the sensitive components of the drone hosted in the central case. The proposed approach is compared to other state-of-the art collision-tolerance strategies and is validated in a $50 \mathrm{~g}$ quadcopter that can withstand high speed collisions.
\end{abstract}

\section{INTRODUCTION}

In recent years, the popularity of drones has continued to grow. Initially intended for professional uses, UAVs (Unmanned Aerial Vehicles) have gradually made their way into people's lives and now serve as very useful tools to assist with tasks that could not, or not as effectively, be achieved by humans or terrestrial robots [1]. While developments in control and sensing have greatly impacted the ease-of-use and versatility of drones, they remain vulnerable to failures [2]. A mistake by the pilot or a missed detection of unforeseen obstacles by a sensor can cause the UAV to collide. The implications of a collision vary considerably depending on the drone's mission, ranging from financial repercussions only, up to more serious effects, such as the inability to find survivors during a search and rescue mission.

Collision resilient drones are specifically designed to withstand severe crashes with obstacles or the ground, without irreversible structural damages. The problem of adequately protecting drones from collisions is tackled with different strategies. A common approach is to add cages or protective structures that shield the sensitive elements of the drone [3][4], such as arms, propellers and central frame. A different strategy involves the use of more complex systems that decouple rotations between the drone and an external cage with a gimbal mechanism in order to avoid the losses of stability of the drone in the event of a collision, therefore preventing it from falling [2]. However, there are still situations where this mechanism can fail, for example while flying in a forest where branches can hamper the free rotation of the protective cage. In addition, correct functioning of the gimbal mechanism requires a stiff cage [2], which is therefore fragile and prone to failure in case of high energy

The authors are with the Laboratory of Intelligent Systems (http://lis.epfl.ch) at École Polytechnique Fédérale de Lausanne (EPFL), CH1015 Lausanne, Switzerland (email: stefano.mintchev@epfl.ch). collisions. To the authors' knowledge, there is no method that prevents impacts from disturbing a platform's stability in all situations and thus prevent a fall to the ground after a collision. Therefore, strategies for protecting the drones from collisions remain crucial to extend their lifespan.

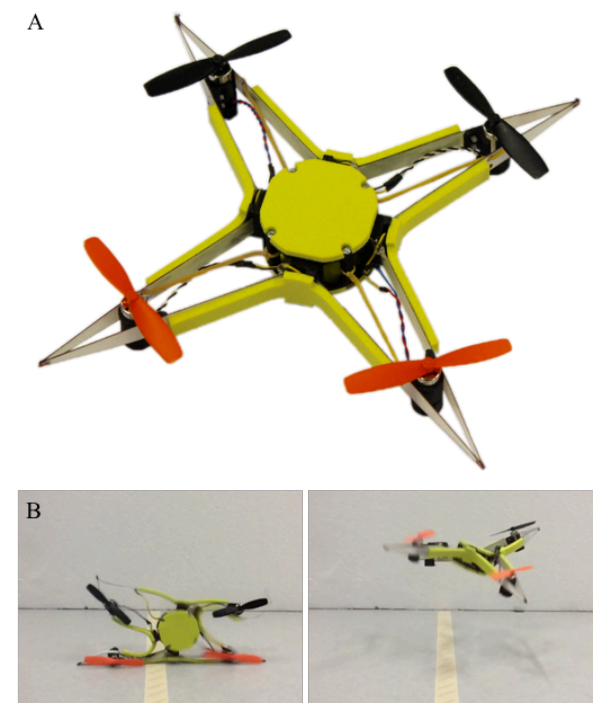

Figure 1. (A) A quadcopter with a crash resilient frame inspired by the wings of insects. (B) Like insect wings, the frame is rigid during flight, but can undergo large deformations without permanent damage during collisions (left pictures), and can automatically reassembly after collision (right picture).

Physical survival is a main priority for all living beings. Therefore, it is not surprising that different solutions to improve physical survivability of artificial systems [5-8] have been inspired by the animal kingdom. Resorting to soft materials is often cited as a viable solution for collision resilience [9][10]. However, a multicopter frame made entirely with soft materials would not withstand required aerodynamic loads and payload weights. In this article, we investigate a new strategy for the development of collisionresilient multicopters that is inspired by a biomechanical solution found in insects. As reported by Mouncastle et al. [11], some insect wings passively deform when colliding with vegetation in order to prevent fast tearing or irreversible structural damages. For instance, wasps have compliant joints (the costal break) embedded in the distal part of their wings that allow them to undergo large but reversible deformations upon collision. Interestingly, their wings are not intrinsically soft, but the stiffness of this joint is tuned such that the wings behave relatively rigidly during flight in order to withstand up to 1000 beats per second, but buckle and undergo large deflections in a collision when forces suddenly increase.

Based on these findings, we propose a dual-stiffness frame for multicopters that effectively withstands collisions. The frame can reversibly transition between a rigid and a soft 
state, combining the advantages of both; stiffness under low loads ensures a stable and efficient flight while mechanical compliance under high loads prevents damages during collisions. Fig. 1A depicts a foldable quadcopter equipped with arms that are rigid within the flight envelope, but fold during a collision (Fig. 1B), successfully avoiding failure due to overloading. This result is obtained by using an intrinsically flexible frame that stiffens when magnetic joints lock it around a central block, which serves as a case for electronics and batteries. During a collision, the magnetic joints behave like "mechanical fuses" that disengage the frame from the central case and let it freely deform without failure. The dual-stiffness frame works in synergy with viscoelastic foam, an energy absorbing material that protects the central case.

First, a discussion of state-of-the-art collision-tolerance strategies for drones and a comparison with the proposed bioinspired approach is presented. Then the design of the collision resilient foldable quadcopter is presented and relevant features and performances are analyzed. A discussion of the results, their scalability, and proposals for future work conclude the paper.

\section{COLlision-TOLERANCE StRATEGIES FOR MUlticopters}

The growing use of drones for ludic and professional applications has fostered the development of different collision-tolerance strategies aiming at minimizing the risk of permanent structural failure. The different strategies for making collision tolerant drones are depicted in Fig. 2. Collision resilient drones exploit structures that limit the force transmitted to the platform's stiff and delicate inner frame and minimize deceleration during a collision. In a multicopter, the sensitive parts that require protection are the central case hosting fragile components, such as batteries control electronics and sensors, and the frame, composed of slender arms that are prone to flexural failure due to their length.

The first approach, which is frequently found in commercial drones, involves planar structures rigidly connected to the arms of the multicopter in order to shield the propellers (Fig. 2A). These structures are usually made of foam, rigid polymers or composite materials. They prevent contact between obstacles and the propellers, therefore avoiding a sudden halt of the rotors and the ensuing destabilization of the drone. In addition, they protect people from the dangers associated with fast spinning propellers. A tight margin is chosen between the propellers and the shielding structures in order to minimize the size and, thus, the weight of the protection. This minimal margin limits the amount of deformation that the protection can withstand. Furthermore, these structures are directly connected to the arms and thus provide limited protection against flexural overloading. Summarizing, this strategy is well suited for shielding the propellers from people and the environment, but is not effective at high speed collisions because of the inability to absorb high energies.

This limitation can be addressed by decoupling the protective structures from the frame with compliant elements or by using flexible arms (Fig. 2B) [12]. The soft elements absorb collision energy, thus limiting the peak forces transmitted to the main frame of the drone. However, those elements make the frame intrinsically soft, and therefore can cause undesired oscillations during flight, inducing losses of stability, slow responses and energetic inefficiency.

To date, the most effective collision-tolerance strategies employ all around structures or cages that can withstand collisions from multiple directions (Fig. 2C). These structures usually exploit long rods of carbon fiber that buckle to absorb energy like Euler springs [3][4]. Composite materials with low density and high strength, such as carbon fiber, can make Euler springs strong and lightweight. However, carbon fiber Euler springs display several shortcomings. Firstly, due to the intrinsic stiffness of carbon fiber, the elements of the protective structures must be long and thin in order to absorb energy without high peak forces. Consequently, these protective structures significantly increase the drone size (by $60 \%$ in [3], e.g.) and thus reduce maneuverability in cluttered environments. A second drawback is that carbon fiber rods have an elastic behavior and do not dissipate energy during deformation. The energy is stored inside the structure and explosively released with a high probability of generating dangerous splinters in case of failure.

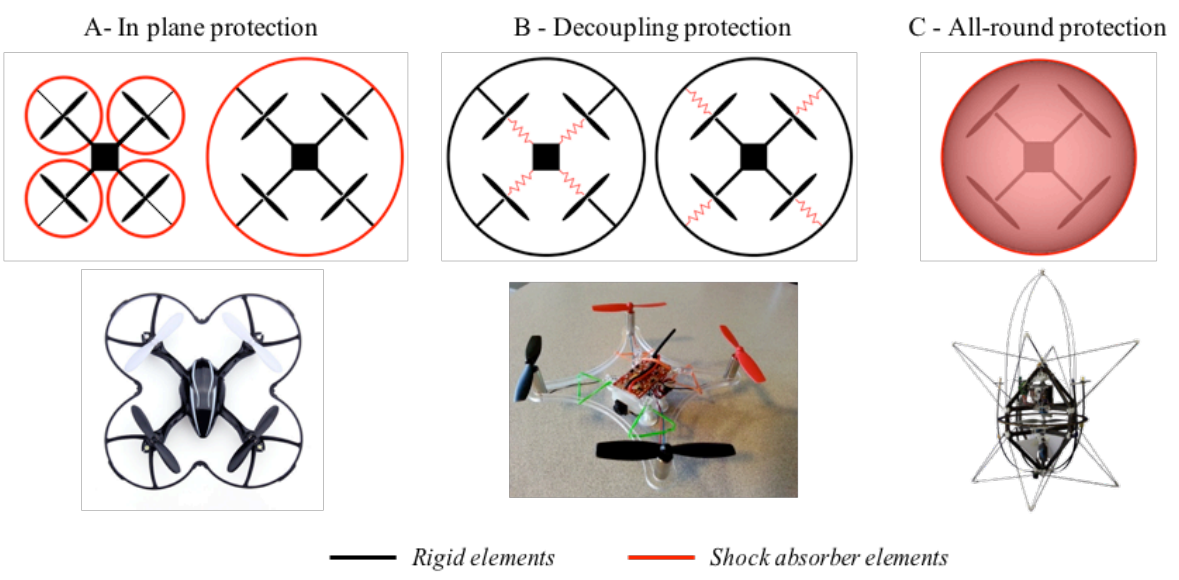

Figure 2. Three collision-protection strategies (top row) for hovering drones with examples (bottom row). (A) Hubsan ${ }^{\circledR}$ X4 with a planar protective frame. (B) The Dream-Catcher, a quadcopter with flexible arms made with elastic bands [12]. (C) The AirBurr with a protective cage based on carbon fiber Euler springs [3]. 


\section{DuAl STIFFNESS FOR COLLISION RESILIENCE}

Flying insects are often subject to collisions, which can happen with vegetation during foraging [13] or against obstacles during flight [14]. An insect needs protection for its body and its slender wings, which suffer from tear and flexural failure. In the majority of insects, body protection is achieved through a sturdy yet flexible exoskeleton that allows the insect to absorb the energy of the impact [15]. On the other hand, only some species of insects evolved specific collision-resilience strategies for protection of their wings.

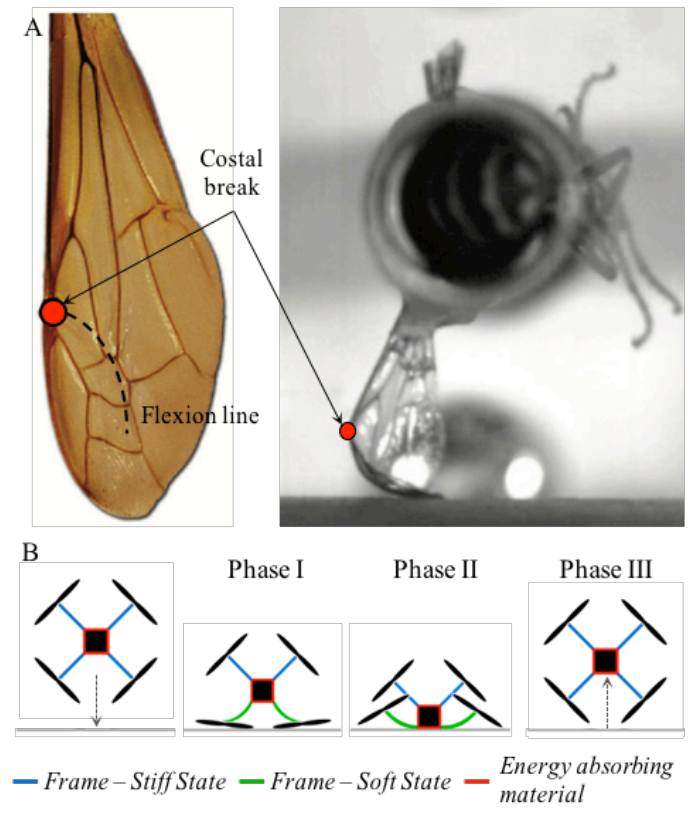

Figure 3. (A) Wasp wings reversibly transition between rigid and soft state by means of flexible joint lines that fold under strong forces (images from [11]). (B) This principle is applied to the mechanical design of quadcopter arms that reversibly transition between stiff (flight mode) and soft (collision mode) states. In phase I, the arms that collide with the ground transition to the soft state and deform without failure. In phase II, the frontal arms are completely deformed and the case hits the ground. The collision is mediated by energy absorbing materials that dissipate most of the kinetic energy of the impact. In phase III, after the collision, the frame recovers its original shape and stiffness without permanent damage.

The wings of insects are composed of stiff cuticle tiles interconnected through flexible joints made of resilin. Cuticle is a biological fiber made of composite material with a load bearing function. It is arranged as a thin membrane reinforced by a complex network of veins. Resilin is a soft elastomeric protein that gives compliance to the wing. The network of cuticle veins makes insect wings rigid enough to withstand intense aerodynamic loads during flight, while regions of resilin allow for small deflections for optimal aerodynamic performances [16]. This intrinsic rigidity could make insects' wings prone to tear or to flexural damages during collisions, with a consequent reduction on flight performance [17][18]. However, Mountcastle et al. [11] found that wasp wings display dual stiffness, that is the ability to reversibly transition between rigid and soft states, which provides mechanical resilience without impairing flight performances. The wings of wasps contain a flexible resilin joint, the costal break, that is often accompanied by a median flexion line (Fig. 3A). This design allows the wing tip to slightly flex during flight (rigid state), but reversibly crumple along the flexion line during collisions (soft state) (Fig. 3B). If the dual-stiffness behavior is impaired, for example by splinting the costal break, the rigid wings undergo severe tear during collision. Therefore, this design provides crash resilience by effectively preventing wing overload during collisions without compromising flight capabilities.

Here we describe a mechanical strategy inspired by the dual stiffness behavior of insect wings that can be used in multicopters for protection of the frame, which is composed of multiple slender arms, and of the central case, which hosts electronics and batteries. The proposed strategy is based on two elements that work in synergy: a frame with dualstiffness properties akin to insect wings and a case protected by energy absorbing materials akin to insects' exoskeletons. As shown in Fig. 3B, a collision entails three main phases: (I) upon contact with an obstacle, the dual-stiffness frame crumples with no permanent damage and dissipates a small amount of kinetic energy, (II) the energy absorbing material mediates the collision between the case and the obstacle, (III) after the collision the dual-stiffness frame bounces back and recovers its original shape and stiffness without permanent damage. The energy absorbing material dissipates the majority of the kinetic energy and contributes to reduce peak decelerations that could cause damages to the components inside the case (e.g. autopilot board, battery and sensors).

The translation of the proposed collision resilience strategy into the design of multicopters is straightforward. The dual stiffness behavior of the arms can be implemented by mainly resorting to two different approaches: elastic instability and load limiter mechanisms. Elastic instability allows slender elastic structures to behave rigidly within a user defined threshold, but reversibly transition to a soft state capable of undergoing large deformations without failure before overloading. Structural instability is exploited by insect's wings [11], but can be scaled up to multicopters as it is mostly correlated to material properties and aspect ratio rather than to absolute length-scales [19]. The foldable boom presented in [20] is an example of load bearing structures that reversibly crumple before overloading and can be adapted to arms of drones of different sizes. Another implementation of dual stiffness frames relies on load limiter mechanisms, the mechanical analogous of "electric fuses" [21][22]: they can rigidly hold the arms together during flight, but release them before overloading (transition to the soft state) during collisions. Load limiter mechanisms can be developed using different technologies (e.g. electromagnetic, pneumatic, elastic snap-fit, or friction based) and can be scaled to different loads. The artificial equivalent of insects' exoskeletons, which mostly function as energy absorbing elements, can be achieved by resorting to protective structures made of materials specifically designed for energy dissipation. Good candidates are visco-elastic foams (e.g. ${\mathrm{D} 3 \mathrm{O}^{\circledR}}^{\circledR}$ and PORON $\mathrm{XRD}^{\circledR}$ ) or microlattice structures [23][24]. These materials have two advantages compared to carbon fiber Euler springs, the elastic structures proposed in literature for energy absorption [3][4]: they are designed to internally dissipate the energy avoiding the issues of explosive release of splinters; and they allow to develop energy absorbing structures that are lighter and more compact. A comparison of weight and size between energy absorbing structures based on PORON XRD ${ }^{\circledR}$ and carbon 
based Euler springs and a discussion of their scalability is presented in Appendix A.

Compared to state-of-the-art strategies (Fig. 2), this bioinspired collision-tolerance design offers several advantages: (i) the rigidity of the frame preserves controllability and efficiency within the flight envelope with respect to what is offered by decoupled protective structures (Fig. 2B.); (ii) the use of specifically engineered materials to absorb energy, for example the visco-elastic foam PORON $\mathrm{XRD}^{\circledR}$, allows to minimize the size and weight (see Appendix A) and improves safety of the protective structures compared to carbon fiber Euler springs; (iii) the structure and layout of the multicopter does not require major changes, as compared to other protective configurations, such as the configuration with coaxial propellers and flaps described in [3], with benefits in terms of design and control simplicity; (iv) foldable arms could allow the drone to be stowed in a small package, a feature that is essential for ease of transportation [25-27].

\section{Design of the Collision RESILIENT QuAdCOPTER}

The bioinspired collision-tolerance strategy requires the implementation of a dual-stiffness frame that rigidly withstands the forces generated by the propellers but folds upon collision, and an additional energy absorbing material to protect the case.

Both the requirements are fulfilled by the quadcopter depicted in Fig. 1. As shown in Fig. 4A, the drone is composed of two main assemblies, a central case and an external frame shaped as a star polygon with four edges. The central case is a single 3D printed part that hosts the autopilot board and the battery. The external frame is composed of fiberglass arms (thickness $0.3 \mathrm{~mm}$ ) held together by four magnetic joints. The motors are connected directly to the external frame. Due to the limited thickness of the fiberglass arms $(0.3 \mathrm{~mm})$, the external frame is extremely soft and can be easily stretched and bent (Fig. 4B). This intrinsic flexibility makes the frame capable of withstanding collisions without being subject to permanent damages.

The desired dual-stiffness behavior of the frame is achieved through four magnetic joints that connects the frame with the case (Fig. 4C and D) and behave like a load limiter mechanism. During flight, the magnets lock the frame around the central case, effectively constraining its deformation, thus ensuring the rigidity of the quadcopter while maneuvering. However, when a force applied to the frame exceeds the flight envelope, the joints disengage, leaving the frame unconstrained, therefore free to deform due to its intrinsic flexibility. Each joint is composed of two spherical magnets in the case and two cylindrical magnets in the connector of the flexible frame (Fig. 4D). The joint is designed in a way that the spherical magnet slightly penetrates inside the connector of the frame (see also Fig. 5A) in order to avoid relative planar motion of the connecting magnets forming the joint. This design, together with the magnetic attraction force, makes the joint a mechanical equivalent of an electric fuse.

The electric wires of the motors are not directly connected to the autopilot board inside the central frame in order to avoid damaging them or hampering the deformation of external frame during collisions (Fig. 4A). Instead, the electric connection is routed through spring-loaded connectors that detach during collisions (Fig. 4D). This design also has the advantage of automatically cutting the power from the motors during collisions, limiting overload of the motors and additional damage to propellers.

The transition to the soft state is reversible and after the collision the drone automatically recovers its original shape and rigidity (Phase III in Fig. 3B). This behavior is ensured by the intrinsic elasticity of the external frame that rapidly recovers its original shape after deformations. In addition, the quadcopter is equipped with soft elastic bands (visible in Fig. 1A and 4C) that connect the central case with each edge of the external frame. The elastic bands, although not significantly hampering the deformation of the frame, foster the rapid reestablishment of the connection between the case and the frame after a collision. This allows the quadcopter to take-off again after most collisions, except when the machine lands upside down.

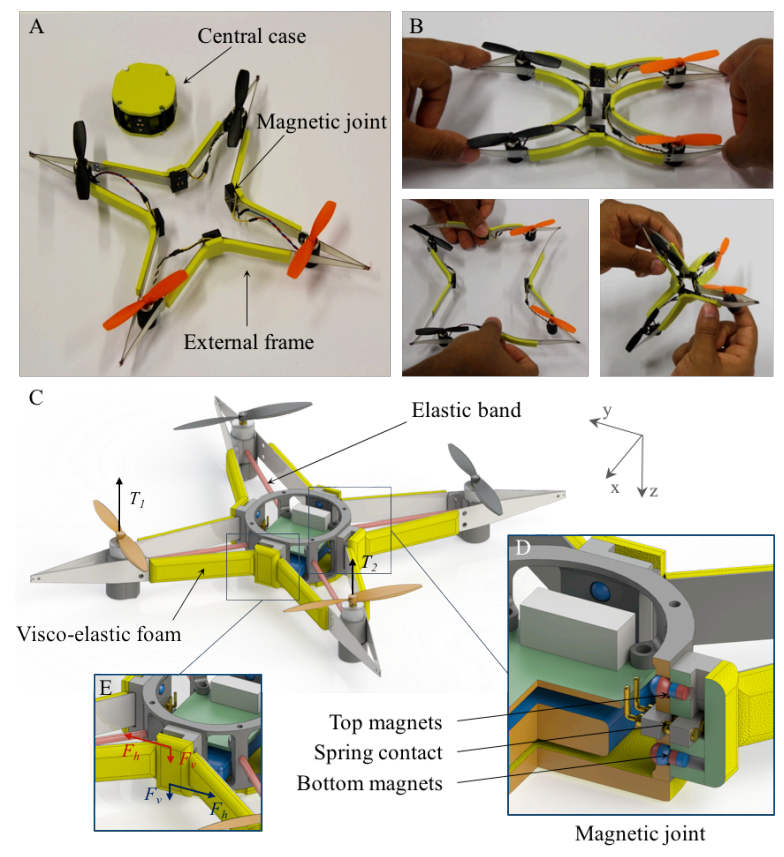

Figure 4. (A) The quadcopter is composed of two main assemblies, the central case and the external frame, connected together by magnetic joints. (B) Intrinsic flexibility of the external frame made with thin fiberglass arms. (C) 3D model of the quadcopter. For sake of clarity, the top protective foam is not shown. (D) Section view to highlight the design of the magnetic joint. (E) Detailed view of the forces in the magnetic joint (see Eq. 1 and 2).

In addition to a dual-stiffness frame, the proposed collision resilient strategy requires additional structures to protect the central case and its content by absorbing the energy from the collision. In the prototype, this feature is achieved by adding 3mm thick layers of PORON 4790-79-09 ShockSeal ${ }^{\mathrm{TM}}$. This visco-elastic foam is placed at the top and bottom of the case, and on the arms in order to protect from frontal and lateral collisions.

\section{EXPERIMENTAL RESULTS}

The quadcopter is equipped with a commercial autopilot board [25] and it is remotely controlled. The energy is provided by a single cell lithium polymer battery (nano-tech $260 \mathrm{mAh}$ ). Overall the quadcopter has a size of $210 \times 210 \mathrm{x}$ 
$26 \mathrm{~mm}$, it weighs 51 grams and has a cost of $\$ 50$. The quadcopter has a flight time of 5 minutes and can carry an additional payload of up to 15 grams. The elements that belong to the collision-tolerance strategy are magnets, foam inserts and elastic bands, with a total weight of 4.66 grams, corresponding to $9 \%$ of the total mass of the drone. In the drones presented in [3] and [4] that exploit all-round protection with Euler springs (Fig. 2C), the percentage of mass corresponding to the protective structures is respectively $14 \%$ and $8 \%$.

The dual stiffness behavior is implemented by using magnetic joints that disengage the external frame from the central case when the applied force overcomes a user defined threshold through the selection of the magnets and of their layout. The experimental characterization of the magnetic joint is illustrated in Fig. 5A. Although each magnetic joint of the drone is composed of two couples of magnets (Fig. 4D), the test involves only a single couple of magnets. The sphere is made of neodymium N35 with a diameter of $3 \mathrm{~mm}$, and the cylinder is neodymium N48 with diameter and height of 2 $\mathrm{mm}$. During the test, the green part of the magnetic joint is constrained, while the yellow one is pulled. The applied force is measured and plotted as function of the displacement (Fig $6 \mathrm{~A})$. The result confirms that the magnetic joint behaves as a mechanical fuse. Indeed, it stays engaged up to a tangential pulling force $F_{\text {Lim }}=1.3 \mathrm{~N}$, which corresponds to a deflection of $0.6 \mathrm{~mm}$. When this threshold is overcome, the two parts of the magnetic joint disengage and freely slide with respect to each other.

To ensure stability of the quadcopter during flight, it is important to verify that each magnetic joint can withstand the forces generated by the propellers without disengaging. With reference to Fig. 5B and Fig. 4E, the tangential forces generated by the magnets in the joint in order to counterbalance the thrust of the propellers are computed with the following equilibrium equations:

$$
\begin{aligned}
& \sum F_{z}=0 \rightarrow 2 F_{v^{-}}-\frac{T_{1}+T_{2}}{2}=0 \\
& \sum M_{x}=0 \rightarrow F_{h} d-b \frac{T_{1}-T_{2}}{2}=0
\end{aligned}
$$

where $F_{v}$ and $F_{h}$ are the vertical and horizontal components of the magnetic force $F_{m}$, and $T_{2}=a T_{1}$, with $a$ varying between 0 and 1 during flight and the thrust achieving a maximum value of $0.32 \mathrm{~N}$. By dividing the values of thrust by two we assume that the force generated by each propeller equally distributes to two adjacent joints. The following inequality must be verified to avoid the disengagement of the magnets in the joint during flight:

$$
\begin{aligned}
& F_{m}=\sqrt{{F_{h}{ }^{2}+F_{v}{ }^{2}}^{2}} \\
& =T_{1} \sqrt{\left(\frac{1+a}{4}\right)^{2}+\left(\frac{b}{d} \frac{1-a}{2}\right)^{2}} \leq F_{L i m}
\end{aligned}
$$

Fig. $5 \mathrm{C}$ shows that, even at maximum thrust $\left(T_{1, M A X}=\right.$ $0.32 \mathrm{~N}$ ), the force required from each magnetic joint during

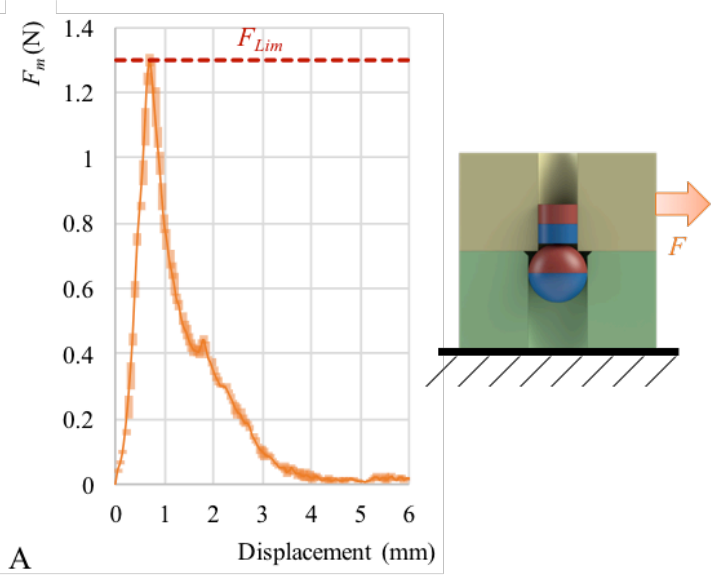

B
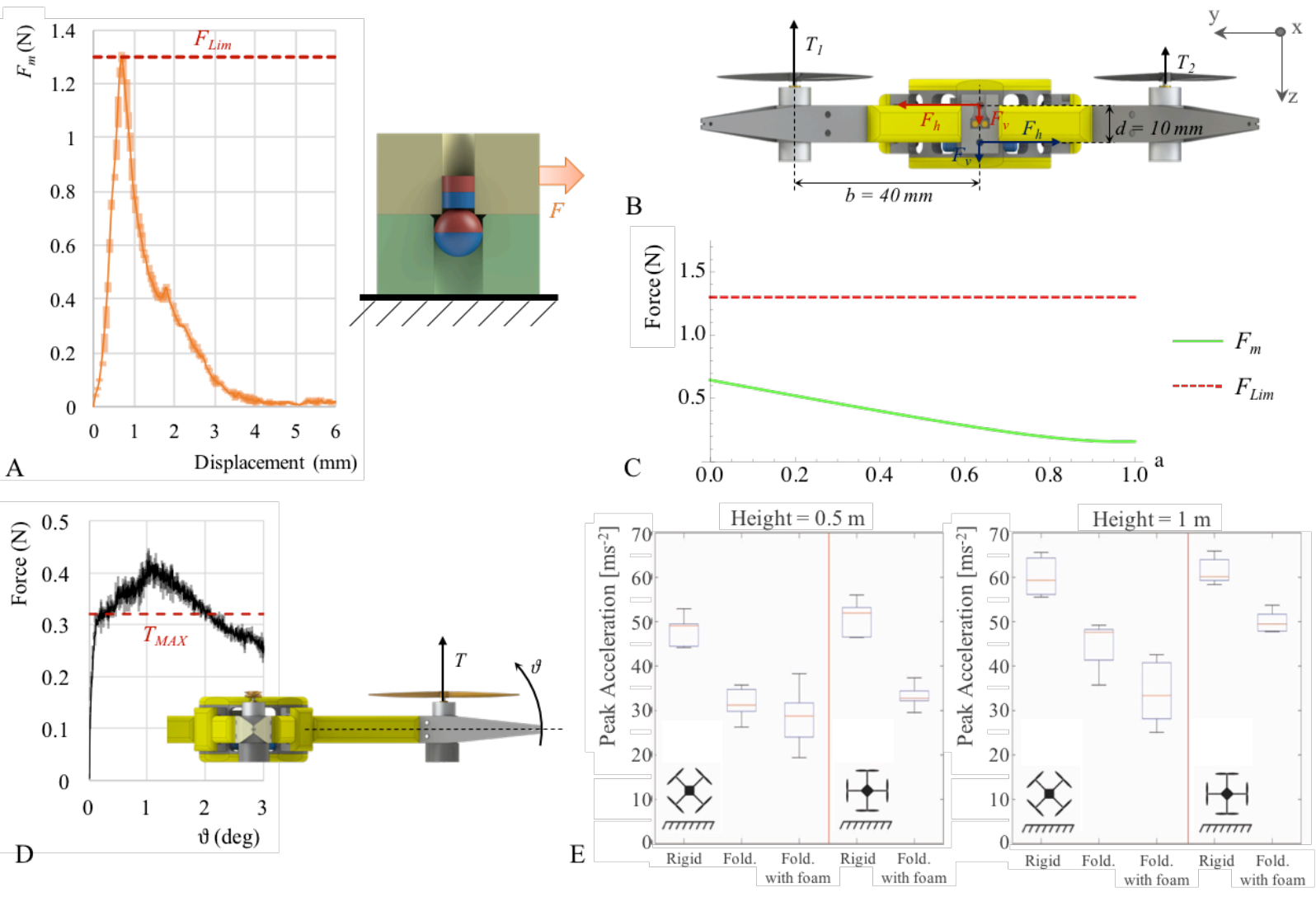

Figure 5. (A) Experimental characterization of the resistive force generated by each couple of magnets in the magnetic joint. (B) Free-body diagram illustrating the forces acting on the magnetic joint during flight. (C) Force acting on each couple of magnets of the magnetic joint in different flight conditions. (D) Experimental characterization of the strength of the arm. (E) Results of drop tests performed on different frames colliding with different orientations. 
the whole flight envelope $(0<\mathrm{a}<1)$ is at maximum half of the disengagement threshold. This ensures that the frame remains stable during flight, even while carrying the maximum payload, with a theoretical safety factor of 2 .

The strength of the frame was measured by pulling one edge of the frame upwards while recording the applied force in order to simulate the effect of the thrust of the propellers (Fig. 5D). The result shows that the frame behaves rigidly with a maximum deflection of $0.2^{\circ}$ at maximum thrust, corresponding to an efficiency loss of just $1 \%$. The disengagement happens at $0.4 \mathrm{~N}$, which corresponds to a safety factor of 1.25 . The experimental value is smaller than the theoretical safety factor of 2 because the fiberglass arms start to twist above $0.3 \mathrm{~N}$, inducing a normal force in the magnetic joints that facilitates their disengagement.

In order to assess the impact acceleration mitigation performance of the bioinspired drone, drop tests were performed under different conditions, with the acceleration of the central case measured using an on-board accelerometer [3]. The bioinspired drone's behavior is compared to that of a classic, rigid-armed drone of same mass for drops from heights of $0.5 \mathrm{~m}$ and $1.0 \mathrm{~m}$. Furthermore, two impact angles were studied. Ten drop tests for each condition were performed. Using parallel vertical wires along which the drone was able to slide in order to ensure the desired orientation at impact, both bioinspired and normal drones were first dropped such that both frontal arms hit the ground simultaneously, thus simulating a head-on collision during flight. During this phase, $3 \mathrm{~mm}$ thick layers of visco-elastic foam were also mounted on the external frame to assess its contribution to energy absorption. Secondly, the drones were dropped such that only one arm hit the ground vertically, thus simulating the worst-case collision scenario, where the energy is directly propagated from the arm to the core of the drone. The results of the drop tests performed from heights of $0.5 \mathrm{~m}$ and $1.0 \mathrm{~m}$ are plotted in Fig. 5E. Both series of drop tests showed the same tendencies in the ability of the bioinspired drone to mitigate the acceleration that is withstood by its central case. The drone equipped with rigid arms yielded very similar accelerations when dropped on its
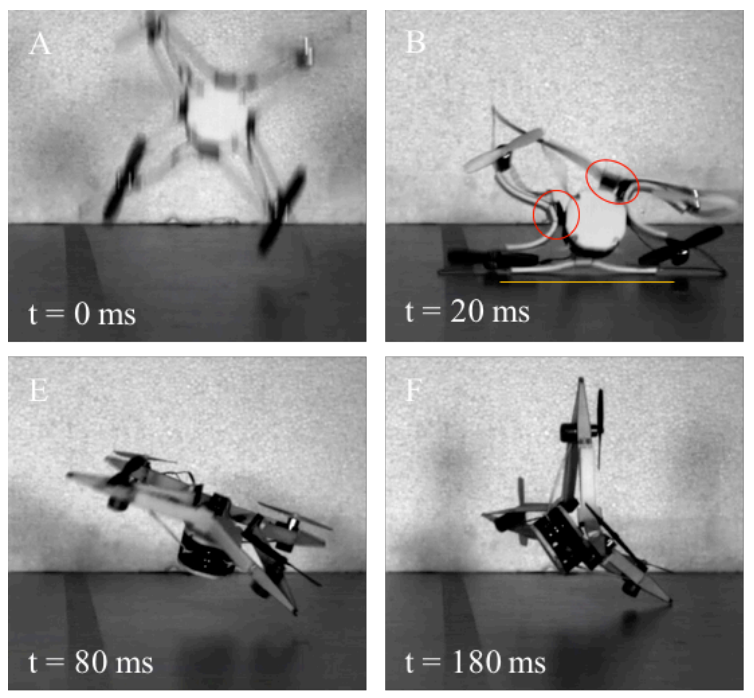

side and on an arm, which underlines the inability of the drone to structurally absorb energy. On the opposite hand, when the tests were performed using the bioinspired collision-resilient quadrotor, a significant decrease in acceleration was observed for both collision orientations. Adding the $3 \mathrm{~mm}$ layers of visco-elastic foam proved to further improve the acceleration mitigation behavior of the bioinspired quadrotor.

An example of head-on collision is shown in Fig. 6, which is composed of multiple snapshots from a video captured with a high speed camera (500 fps). The quadcopter is released from $2 \mathrm{~m}$ and collides against the floor. Immediately after the collision, the magnetic joints disengage and the external frame undergoes large deformations thanks to the intrinsic flexibility of the thin fiberglass arms. This is clearly visible in Fig. 6A where the deceleration triggers the disengagement of three joints (see red circles) and leaves the frame free to deform. It is also interesting to note that the collision between the central case and the floor is mediated by the visco-elastic foam placed in the front of frame (see yellow line). The foam acts as a cushion and contributes to mitigating the deceleration experienced by the case and its components. After the collision, the quadcopter bounces back in the air (Fig. 6C). Now, the four joints are all disengaged and the central case is constrained to the deformed frame by the elastic bands that prevent the separation of the two components (Fig. 6D, E and F) and help to restore the original shape of the drone when the collision has ended (Fig. $6 \mathrm{H})$. It is also interesting to note that the external frame undergoes severe out of plane deformation, which is visible in Fig. 6F. The reader is encouraged to refer to the video attached to this paper for a better understanding of the collision sequence.

\section{DISCUSSION}

The uniqueness of the proposed design lies in the fact that the frame is rigid during flight, but softens during collisions. This allows combination of the advantages of both rigid and soft systems: stability and rapid response to user commands during flight, leading to flight performance

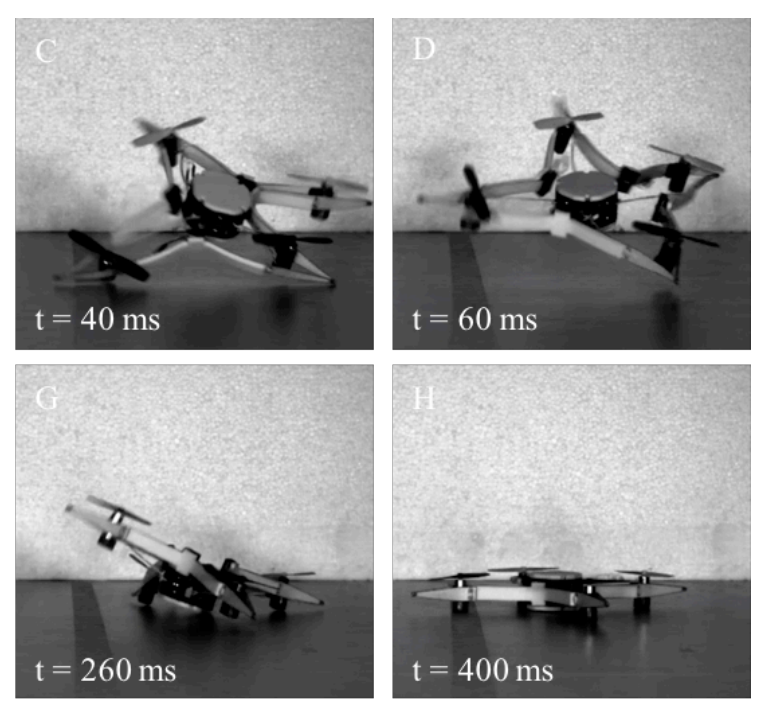

Figure 6. Snapshots of a frontal collision, from contact with the floor (A) to the end of the process $(\mathrm{H})$. The reader is encouraged to refer to the attached video. 
equivalent to a drone equipped with a standard rigid frame, and crash resilience like a soft system. The experiments showed a satisfying survivability of the frame of the drone, that withstood roughly 50 collisions with no permanent damage. On the other hand, the propellers failed twice during the tests due to bending. Although propellers are an affordable component, a failure still affects the capability of the drone to fly again. As mentioned in Section II, this issue can be further mitigated by adding local protections around the propellers, similar to the solution proposed in Fig. 2A.

Another important topic concerns the scalability of the proposed strategy. The contact force generated by permanent magnets scales unfavorably at large scales [28]. Therefore, the design of the dual-stiffness frame for larger drones should resort either to snap-fit locking mechanisms or to elastic structures that undergo elastic instability, which are both scalable solutions that are correlated to material properties and the aspect ratio rather than to absolute length-scales [19]. Also, we show in Appendix A that the protective foam is a scalable solution for energy absorption. Indeed, Fig. 7D shows that the mass of the foam increases linearly with the mass of the drone that needs to be protected. On the other hand, in larger drones, propellers are more sensitive to damage due to their higher kinetic energy. Therefore, local protection mechanisms or foldable propellers become recommended to increase the chance of surviving collisions.

Finally, it is worth noticing that the external frame shaped as a star polygon can be easily adapted to implement different types of multicopters by using a star polygon with additional edges, for example six for hexacopters and eight for octocopters.

\section{CONCLUSIONS}

Physical survival is fundamental for living beings and it becomes necessary also for robots whenever they operate into an unstructured environment. This study demonstrates the feasibility of achieving the physical survivability of a quadcopter using a biomechanical strategy evolved by insects. Crash resilience is obtained without resorting to intrinsically soft structures that would compromise stability and efficiency during flight, but rather with a dual-stiffness frame that softens during collisions. This approach allows to combine the benefits of both rigid and soft materials into a single device increasing its versatility and functionality. Therefore, this solution is also interesting for other mobile robots or manipulators that cannot resort to intrinsically soft materials due to the necessity of generating high forces during locomotion or grasping, but need to soften to mitigate damage from collisions or for safe interaction.

\section{APPENDIX A}

The bioinspired strategy exploits specifically engineered energy absorbing materials that are more compact and lighter than carbon fiber Euler springs. For a comparative study, we consider a collision with the ground of a mass $M$ that falls from the defined height $h$ (Fig. 7). We study two energy absorbing structures: a visco-elastic foam (PORON 4790-7909 ShockSeal ${ }^{\mathrm{TM}}$ ) with a cylindrical shape (radius $R_{F}$, length
$L_{F}$ and density $\sigma_{F}$ ) and an Euler rod made of carbon fiber (radius $R_{E}$, length $L_{E}$ and density $\sigma_{E}$ ). The protective structure is in charge of dissipating the potential energy of the drone, ensuring a deceleration below a user defined threshold $\left(a_{M A X}\right)$, that is often given by the intrinsic resistance of the main components of the drone (e.g. electronics boards or sensors).

Fig. 7D illustrates a comparison of length, mass and radius of the two protection systems as function of the mass $M$ for a drop from $1 \mathrm{~m}$ high and a maximum deceleration $a_{M A X}=1000 \mathrm{~m} / \mathrm{s}^{2}$. The results confirm that carbon fiber Euler springs are in general longer and heavier than foam-base protective structures, thus compromising the size and the flight time of the drone. Furthermore, Euler springs have a smaller radius, causing a concentration of the collision force on a small volume, thus increasing the risk of piercing. Finally, the graphs highlight that both approaches are scalable with respect to the mass of the drone. The same results can be achieved considering a fixed mass of the drone but varying heights.
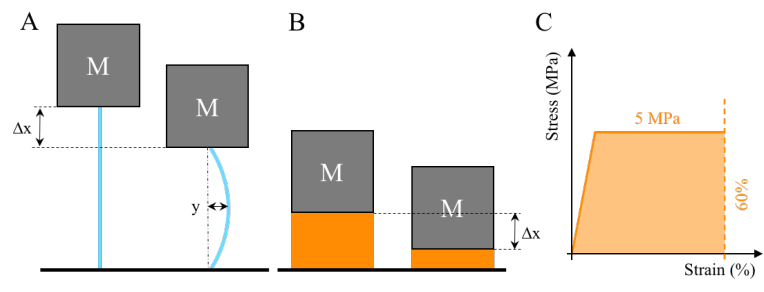

Geometry to absorb $1 \mathrm{~m}$ fall

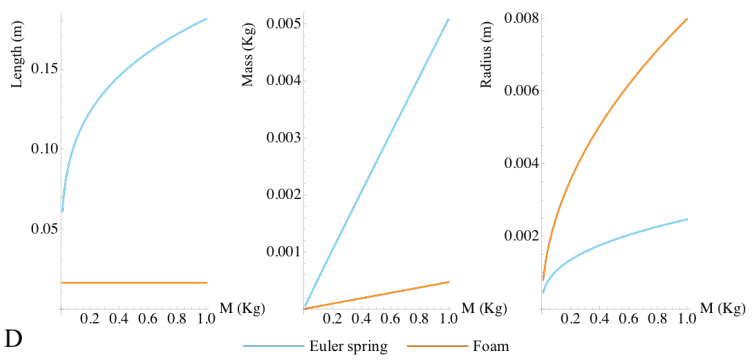

Figure 7. Comparison between Euler springs visco-elastic foam for energy absorption for a drop from $1 \mathrm{~m}$ high. (A) and (B) Schematics of deformations due to a collision. (C) Compression force deflection (CFD) curve of PORON 79-09 at strain rates of 2000 /s (high speed collision). (D) Results of the comparison.

The plots of Fig. 7D are obtained using the following method. The energy of the impact is:

$$
U(h, M)=M g h
$$

Assuming constant deceleration during the impact, the minimum deformation of the protection that ensures a deceleration below the user defined threshold is:

$$
\Delta x(h, a)=\frac{g h}{a_{M A X}}
$$

As illustrated in Fig. 7C, during a high speed deformation the foam compresses up to a strain $\varepsilon=0.6$ with an almost constant stress $\sigma=5 \mathrm{MPa}$. Therefore, the foam can absorb an energy per unit of volume that can be approximated as $\sigma \varepsilon$. The length of the foam can be computed considering a conservative value of maximum strain of 0.6 : 


$$
L_{F}(h, a)=\frac{\Delta x(h, a)}{0.6}
$$

The radius of the foam is computed considering the amount of energy that the material can absorb:

$$
R_{F}(h, a, M)=\sqrt{\frac{U(h, M)}{\pi \sigma \Delta x(h, a)}}
$$
is:

Finally, the mass of the foam based protective structure

$$
M_{F}(h, a, M)=\sigma_{F} \pi R_{F}^{2} L_{F}
$$

The weight and size of the Euler carbon rod can be optimized assuming the energy absorption up to failure, when the internal stress in the rod reaches the yield limit $\left(Y_{S}\right)$. During a collision, the carbon rod deflects by $(y)$ under the action of an axial force $(F)$ :

$$
\begin{aligned}
& y(h, a)=0.9 L \sqrt{\frac{E J}{L^{2} F_{c r}} k \frac{\Delta x(h, a)}{L}} \\
& F(h, a)=F_{c r}+\frac{E J}{L^{2}} k \frac{\Delta x(h, a)}{L}
\end{aligned}
$$

where $J$ is the polar moment of inertia of the area section, $E$ is the Young Modulus, $F_{c r}$ is the critical buckling force of the rod and $k=8.09$ [3].

The radius and length of the rod can be computed by solving the following system:

$$
\left\{\begin{array}{c}
U(h, a, M)=E J \frac{\Delta x(h, a)}{L^{2}}\left(\pi^{2}+\frac{k}{2} \frac{\Delta x(h, a)}{L}\right) \\
Y_{S}=\frac{F(h, a) y(h, a)}{J} R_{E}
\end{array}\right.
$$

\section{ACKNOWLEDGMENT}

This work was supported by the Swiss National Science Foundation through the National Centre of Competence in Research Robotics (NCCR Robotics).

\section{REFERENCES}

[1] D. Floreano and R. J. Wood, "Science, technology and the future of small autonomous drones," Nature, vol. 521, no. 7553, pp. 460-466, 2015.

[2] A. Briod, P. Kornatowski, J.-C. Zufferey, and D. Floreano, "A Collision-resilient Flying Robot," Journal of Field Robotics, vol. 31, no. 4, pp. 496-509, Jul. 2014.

[3] A. Klaptocz, A. Briod, L. Daler, J. C. Zufferey, and D. Floreano, "Euler spring collision protection for flying robots," in IEEE International Conference on Intelligent Robots and Systems, 2013, pp. 1886-1892.

[4] Y. Mulgaonkar, G. Cross, and V. Kumar, "Design of small, safe and robust quadrotor swarms," in Proceedings - IEEE International Conference on Robotics and Automation, 2015, vol. 2015-June, no. June, pp. 2208-2215.

[5] S. Mintchev and D. Floreano, "Adaptive Morphology: A Design Principle for Multimodal and Multifunctional Robots," IEEE Robot. Autom. Mag., vol. 23, no. 3, pp. 42-54, 2016.

[6] W. Yang, I. H. Chen, B. Gludovatz, E. A. Zimmermann, R. O. Ritchie, and M. A. Meyers, "Natural Flexible Dermal Armor," Adv. Mater., vol. 25, no. 1, pp. 31-48, 2013.

[7] A. Bührig-Polaczek, C. Fleck, T. Speck, P. Schüler, S. F. Fischer, M. Caliaro, and M. Thielen, "Biomimetic cellular metals-using hierarchical structuring for energy absorption," Bioinspir. Biomim., vol. 11, no. 4, p. 45002, 2016.
[8] A. K. Stowers and D. Lentink, "Folding in and out: Passive morphing in flapping wings," Bioinspiration and Biomimetics, vol. 10, no. 2, pp. 1-16, 2015.5, 2014.

[9] D. Rus and M. T. Tolley, "Design, fabrication and control of soft robots," Nature, vol. 521, no. 7553, pp. 467-475, May 2015.

[10] N. W. Bartlett, M. T. Tolley, J. T. B. Overvelde, J. C. Weaver, B. Mosadegh, K. Bertoldi, G. M. Whitesides, and R. J. Wood, "A 3Dprinted, functionally graded soft robot powered by combustion," Science (80-. )., vol. 349, no. 6244, p. 161 LP-165, Jul. 2015.

[11] A. M. Mountcastle and S. a Combes, "Biomechanical strategies for mitigating collision damage in insect wings: structural design versus embedded elastic materials," J. Exp. Biol., vol. 217, no. December, pp. 1108-1115, 2014.

[12] Available: http://www.rcgroups.com/forums/showthread.php?t=2253209. [Accessed: 24- August - 2016]

[13] D. J. Foster and R. V Cartar, "What causes wing wear in foraging bumble bees?," The Journal of experimental biology, vol. 214, no. Pt 11, pp. 1896-1901, 2011.

[14] A. K. Dickerson, P. G. Shankles, N. M. Madhavan, and D. L. Hu, "Mosquitoes survive raindrop collisions by virtue of their low mass.," Proc. Natl. Acad. Sci. U. S. A., vol. 109, no. 25, pp. 9822-7, 2012.

[15] S. Gunderson and R. Schiavone, "The insect exoskeleton: A natural structural composite," JOM, vol. 41, no. 11, pp. 60-63, 1989.

[16] A. M. Mountcastle and S. A. Combes, "Wing flexibility enhances load-lifting capacity in bumblebees," Proceedings of the Royal Society of London B: Biological Sciences, vol. 280, no. 1759, Mar. 2013.

[17] S. A. Combes, J. D. Crall, and S. Mukherjee, "Dynamics of animal movement in an ecological context: dragonfly wing damage reduces flight performance and predation success," Biol. Lett., vol. 6, no. 3, pp. 426-429, May 2010.

[18] A. Hedenström, C. P. Ellington, and T. J. Wolf, "Wing wear, aerodynamics and flight energetics in bumblebees (Bombus terrestris): an experimental study," Funct. Ecol., vol. 15, no. 4, pp. 417-422, 2001.

[19] P. M. Reis, "A Perspective on the Revival of Structural (In)Stability with Novel Opportunities for Function: From Buckliphobia to Buckliphilia," J. Appl. Mech., vol. 82, no. 11, p. 111001, Sep. 2015.

[20] J. Block, M. Straubel, and M. Wiedemann, "Ultralight deployable booms for solar sails and other large gossamer structures in space," Acta Astronaut. (U.K.), vol. 68, no. 7-8, pp. 984-992, 2011.

[21] A. J. Flocchini, "Mechanical fuse," U.S. Patent 4 716 635, 1988.

[22] M. Plooij, G. Mathijssen, P. Cherelle, D. Lefeber, and B. Vanderborght, "Lock your robot: A review of locking devices in robotics," IEEE Robot. Autom. Mag., vol. 22, no. 1, pp. 106-117, 2015.

[23] Y. Liu, T. A. Schaedler, and X. Chen, "Dynamic energy absorption characteristics of hollow microlattice structures," Mechanics of Materials, vol. 77, pp. 1-13, 2014.

[24] A. G. Evans, M. Y. He, V. S. Deshpande, J. W. Hutchinson, A. J. Jacobsen, and W. B. Carter, "Concepts for enhanced energy absorption using hollow micro-lattices," International Journal of Impact Engineering, vol. 37, no. 9, pp. 947-959, 2010.

[25] S. Mintchev, L. Daler, G. L'Eplattenier, L. Saint-Raymond, and D. Floreano, "Foldable and self-deployable pocket sized quadrotor," in Robotics and Automation (ICRA), 2015 IEEE International Conference on, 2015, pp. 2190-2195.

[26] L. Dufour, K. Owen, S. Mintchev, and D. Floreano, "A drone with insect-inspired folding wings," in 2016 IEEE/RSJ International Conference on Intelligent Robots and Systems (IROS), 2016, pp. $1576-1581$.

[27] S. Mintchev and D. Floreano, "A pocket sized foldable quadcopter for situational awareness and reconnaissance," 2016 IEEE International Symposium on Safety, Security, and Rescue Robotics (SSRR). pp. 396-401, 2016.

[28] J. S. A. and D. P. Arnold, "A study of scaling and geometry effects on the forces between cuboidal and cylindrical magnets using analytical force solutions," J. Phys. D. Appl. Phys., vol. 41, no. 10, p. 105001, 2008. 